\title{
Probing X-ray irradiation in the nucleus of NGC 1068 with observations of high-J lines of dense gas tracers (Erratum)
}

J. P. Pérez-Beaupuits ${ }^{1}$, M. Spaans ${ }^{1}$, F. F. S. van der Tak ${ }^{2,1}$, S. Aalto ${ }^{3}$, S. García-Burillo ${ }^{4}$, A. Fuente ${ }^{4}$, and A. Usero ${ }^{4}$

1 Kapteyn Astronomical Institute, University of Groningen, Landleven 12, 9747 AD Groningen, The Netherlands e-mail: jp@astro.rug.nl

2 SRON Netherlands Institute for Space research, Landleven 12, 9747 AD Groningen, The Netherlands

3 Onsala Rymdobservatorium, Chalmers Tekniska Högskola, 43992 Onsala, Sweden

4 Observatorio Astronómico Nacional, C/ Alfonso XII 3, 28014 Madrid, Spain

A\&A 503, 459-466 (2009), DOI: 10.1051/0004-6361/200912350

Key words. galaxies: ISM - galaxies: individual: NGC 1068 - galaxies: Seyfert - ISM: molecules: - radio lines: ISM radio lines: galaxies - errata, addenda

An error occurred during the final processing of the publication of Fig. 1. The first spectrum of Fig. 1 has been published four times, replacing the three other spectra. The correct Fig. 1 is published on the next page. 

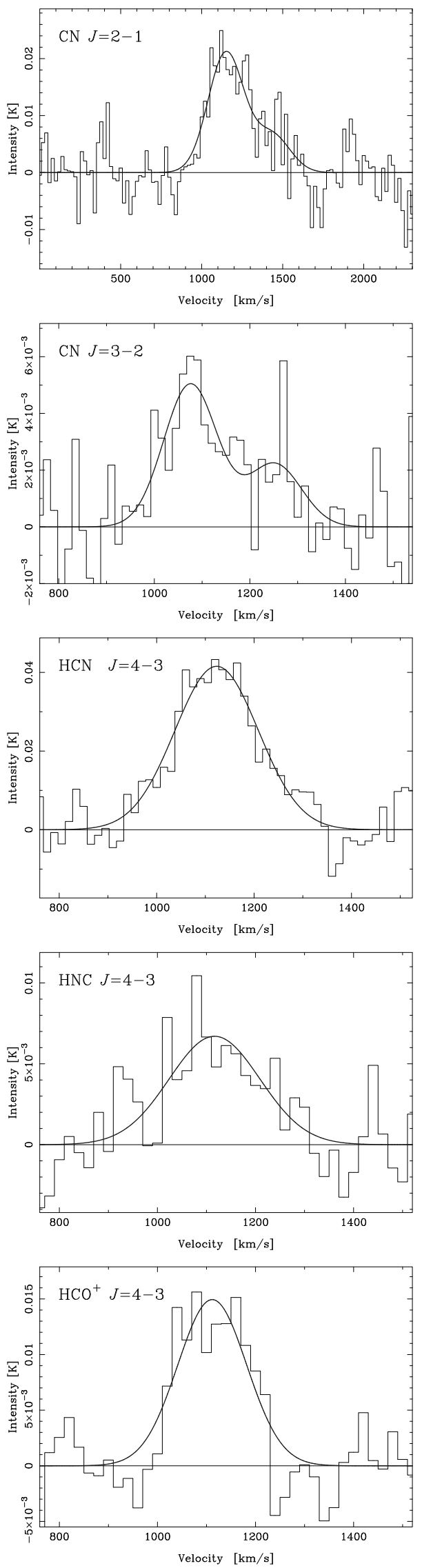

Fig. 1. Molecular line emissions in NGC 1068. The velocity resolution was reduced to $20 \mathrm{~km} \mathrm{~s}^{-1}$ for all the lines. The spectra are centered with respect to the heliocentric systemic velocity $v_{\mathrm{sys}}=1137 \mathrm{~km} \mathrm{~s}^{-1}$. 